\title{
Physiology of The Dependence on Chemical And Psychogenic Psychoactive Factors
}

\author{
Baitubaev DG*1 and Baitubaeva $\mathrm{MD}^{2}$ \\ ${ }^{1}$ Dispensary of Health Department of the East Kazakhstan region, Kazakhstan \\ ${ }^{2}$ Assistant-lecturer of the Semen State Medical University, Kazakhstan \\ *Corresponding author: Baitubaev DG, Dispensary of Health Department of the East Kazakhstan region, Kazakhstan \\ To Cite This Article: Baitubaev DG. Physiology of The Dependence on Chemical And Psychogenic Psychoactive Factors. Am J Biomed Sci \& Res. 2019 \\ - 5(5). AJBSR.MS.ID.000959. DOI: 10.34297/AJBSR.2019.05.000959.
}

Received: 眥 June 25, 2019; Published: 眥 October 17, 2019

\begin{abstract}
The work shows the role of the autonomic nervous system in functioning of long-term memory, the identity of functioning of the mechanisms of long-term memory in the evolutionary adaptation of a man and dependence on psychoactive substances. It is shown that the dependences of the body on psychoactive substances are the states of progredient adaptation, that the states of dependence of the organism on psychoactive substances and on psychogenic psychoactive factors are the states of the same type. Classification of psychoactive factors is given. It is proposed to create a new branch of medicine combining study of the body's dependence both on chemical and psychogenic psychoactive factors. Onomastic definitions to be used in this new branch of medicine are presented.
\end{abstract}

Keywords: Hypermnesia, Engrams of euphoria or any other psychotropic effect; Desirable from the point of view of the consumer; A psychotropic effect in the long-term memory

\section{Urgency of the Issue}

Identification and increase in the number of the organism's dependencies on various external factors of psychogenic origin makes us wonder whether they have the same biological mechanism of occurrence as the body's dependence on chemical psychoactive substances. And whether these states are states of the same type?!

Purpose and objectives of the study. Hypermnesia as an integral part of the mechanism of human evolutionary adaptation. The role of the autonomic nervous system in long-term memory performance

It is a well-known fact that in long-term memory of a person, the effects of external factors remain longer, if they caused strong emotional reactions. Emotional memory is formed very quickly and often from the first time (unlike conditional memory). This phenomenon played an important role in the survival and evolutionary development of human. Memorizing such a psychogenic factor as a predator's attack, accompanied by pavor, allowed to avoid predators in the future. Memorizing situations accompanied by positive emotions - meeting the food needs, influence of heat - contributed to seeking the influences beneficial for the body in the future.

The leading role in the memory formation is played by the limbic system. But studying the mechanisms of long-term memory functioning reveals the instant dependence of the strength of fixation of some external influences in memory on the severity of the emotional responses accompanying those effects. This fact make us to pay attention to the role of the autonomic nervous system (ANS). Indeed, one of the main components of emotional responses are the responses of the autonomic nervous system showing as various bodily sensations: changes in the heart rhythm, increase or decrease in muscle tone, etc.

According to a hypothesis of the Canadian scientist Hebb [1], the transition of information from short-term to long-term memory occurs due to the formation and fixation of very complicated, stable structural chemical changes at the systemic, synoptic, and cellular levels. This leads to consolidation of neurons with high synaptic conductivity and formation of memory engrams fixing the external 
situation and the subject's attitude to it. When speaking about the regulation of synaptic efficiency, attention is paid primarily to the systems of biologically active substances serving as intermediate agents in synaptic transmission, to factors providing modulation of the efficiency of synaptic transmission and the long-term preservation of these shifts in the neural networks.

So, Kendel (1986) [1], Kruglikov (1986) [1], E.G. Gromova (1980) [1] in their studies have established that acetylcholine affects the synthesis of new receptor molecules or the unmasking and activation of already existing receptor proteins, the sensitivity of cortical neurons increasing up to recording the trace in the memory. Noradrenergic mechanisms of the brain provide the creation and temporary preservation of multineuronal constellations - engrams, the serotonergic system accelerates the learning process, facilitates fixation of engrams, and elongates the skill retention. Long-term memory processes are affected by GABA and glutamic acid. According to G. Ungar (1977) [1], I.P. Ashmarin (1987) [1], neuropeptides together with mediators, create specific receptor mosaic sets on the postsynaptic membrane that facilitates the rapid conduction of a certain type of excitation. Adrenocorticotropic hormone (ACTH) and corticosteroids, endogenous opioids - endorphins and enkephalins - have a pronounced effect on learning and memory. Thus, the "keeper" for all forms of neurological memory is the system of interneuronal interactions, and the participation of neurotransmitters, neuropeptides, information biopolymers consists in their influence on the quality of these interactions, their fixation. That is, the neurotransmitters released by the ANS in response to an external stimulus are a sort of modulators of transition and fixation of information in long-term memory.

Identity of the mechanisms of hypermnesia of psychoemotional effects under the influence of psychoactive substances to the evolutionary adaptive mechanism of hypermnesia

Memory is a constantly functioning neurodynamic system. The effects of any external factor regardless its nature - psychogenic or chemical, causing positive, favorable shifts in the person's psychophysical well-being are recalled more often, and a person subconsciously seeks to re-experience the effect of this factor. Therefore, the engrams of positive emotions serve as stigma-attitudes resulting in the person's adjusting behavior. They serve as a starting point for the beginning of the psychic dependence of the body on the external factor that caused a positive psychophysical shift in a person. The repeated influence of an external psychoactive factor contributes to a greater psychic stigmatization of a person. It is noticed that during the influence of the psychoactive factor, the sensations caused by the influence of the accompanying stimuli without psychoactive properties are also fixed at the same time. This indicates that the overall hypermnesticity of the brain increases under the influence of the psychoactive factor. Therefore, engrams of the emotional memory of euphoria, are able in the future to be stimulated by the influence of concomitant stimuli too - the environment in which the psychoactive factor was active, etc. As humanity reveals that positive (from the point of view of the consumer) emotions can be caused by the use of alcohol, opium, marijuana, tobacco, this has led not only to the increase in the habitual, regular use of these substances, but also to the awareness of the fact that it can cause dependence and affect not only the consumer of these substances, but also the society at large. These substances were classified under the general name - psychoactive substances (abbreviated as PAS).

The development of industrial production of alcohol and the chemical industry, the increase in the number of people suffering from dependence on various psychoactive substances, considering the dependence on psychoactive substances as a disease, the need for studying and treating such dependences, all this resulted in the creation of a medical branch - narcology.

Object and methods of investigation: Vegetotropic properties of psychoactive substances. Physiology of progredient adaptation. Mechanisms of fixation of the effects of psychogenic psychoactive factors in long-term memory

Almost all dependence-producing psychoactive substances turned out to have vegetotropic properties - primarily, they affect VNC receptors, causing the release of neurotransmitters. As it was mentioned above, neurotransmitters modulate the preservation of psychoemotional responses in long-term memory after the influence of these substances. "Catalyzing" role of neurotransmitters in long-term memory functioning can be confirmed by the fact that people with different types of dependence remember their feelings of the first episode of smoking, drinking alcohol, drugs even in decades. And it turned out that the more pleasant the subjective effect under the influence of a substance is and the more pronounced vegetative responses brightening the emotions are, the stronger the fixation of these sensations in long-term memory is and the higher the rate of occurrence of dependence is. This phenomenon is designated as narcogenicity of the substance. So, psychoactive substances with high narcogenicity - opiates - cause the accelerated development 
of dependence. But under the influence of substances with low narcogenicity, the occurrence of dependence requires the abuse period.

One of the urgent issues of medicine is the explanation of the biological mechanism of increasing resistance in a PAS-dependent person. No matter how accurately scientific research explain qualitative changes at the cellular, molecular level, leading to an increase in resistance in a PASdependent person - these changes are obviously to have nondamaging, adaptive nature, otherwise they wouldn't lead to the increased resistance. And according to the dialectical principle of the mutual transition of qualitative changes to quantitative ones, the accumulation of these changes should lead to qualitative and quantitative changes in the system responsible for the adaptation of the body as a whole - in the neuroendocrine system. A new field in the physiology of adaptation - progredient adaptation, discovered [2], based on the ability of the neuroendocrine system to hypertrophy under regular PAS exposure, allowed validating PASdependences not as diseases, but as states of progredient adaptation.

After all, in the 30s of the last century, PK Anokhin described the phenomenon of "advanced excitation", when the neuroendocrine system, in response to an external stimulus, makes an excessive release of neurotransmitters, hormones and take a pause for self-restoration, during which the assimilation-restoration processes in the endocrine system dominate over processes of dissimilation. The regular predominance of assimilation processes over the processes of dissimilation under the PAS exposure leads to the hypertrophy of endocrine system. Histological evidence is the Selje's stress study: "the adrenals bloom" the adrenal medulla - the modified sympathetic ganglion [3]- which during a pause, recovers and avoids exhaustion. The adrenal cortical zone, producing hormones adaptationcorticosteroids, is hypertrophied. Also, another urgent issue of medicine is the validation of the physiological process resulting under the influence of increasing doses of a psychoactive substance and the response increase in body tolerance.

Hypertrophy of the endocrine system leads to the direct growth of the adaptive capabilities of the organism relative to the exposure dose growth, and every subsequent, potentially pathogenic, extreme dose "is met" by the hypertrophied, hyperproductive endocrine system and the dose has already subextreme - not a pathogenic - effect. That is, on each floor of adaptation, under the influence of a new increased dose of PAS, the body primarily experiences stress with orientation and resistance phases. But later the stage of depletion does not occur, since earlier due to extra output of neurotransmitters, the endocrine system "took pauses" during which assimilation processes dominated over dissimilation processes and hypertrophy of the endocrine system occured. So, the endocrine system is ready again to protect the body against the larger dose of PAS. The states of regular stress and chronic stress differs in pauses. It is conceivable that stress, whatever regular it may be, without depletion phase is not a disease. Also, while under the influence of normal pathogenic factors without vegetotropic properties first some tissue is damaged, and only after that the ANS produce a protective response, PASs, in contrast, affects primarily the receptors of the ANS, due to their vegetotrophy. This results in the timely high-level protective response of the body, preventing damage.

Endocrine system hypertrophy, and therefore its high adaptive sufficiency, not only prevents damage, but also eliminates the need to compensate for the adaptive capabilities of the body at the expense of some tissues, systems of the body, so the disease does not develop. ANC productivity due to compensatory, excessive release of neurotransmitters explains adrenergic tension in deprivation syndrome, too. Productivity of the sympathetic part of the ANS against the background [4] of the gradual exhausion of the parasympaticus (the adrenergic system is more stable in ontogenesis, too) explains - according to "the age" of narcotism - the transformation of initial sedative effect of hypnotics, alcohol and opiates into their stimulating effect. In the final stages of PAS dependence, depletion of the adaptive capabilities of the organism (the receptors of the protective systems alarm about it) leads to a concurrent decrease in the PAS doses tolerated by the dependent person, the body being not damaged repeatedly. Any pathology in PAS dependence is an associated event.

Over time it has been observed, that the dependence can occur in a person under the influence of psychogenic factors too: people who committed bad sexual crimes can develop overanxiety to repeat them, the state of falling in love results in affection and attraction to the object of love. These situations, as well as in PAS dependence, are accompanied by strong emotions and vegetative responses: fear and excitement in case of crime, a feeling on top of the world in case of love, etc. They provide fixation of the effects of these psychogenic psychoactive factors (PAFs) in long-term memory. Also, like in PAS dependence, vegetative responses under the influence of psychogenic PAFs increase the overall hypermnesticity of the brain - those who commit serial sexual crimes remember in detail every crime they commit, the lovers remember their first meeting, etc. With the development of civilization, 
a lot of psychogenic factors capable of causing dependence emerged. These are ideological psychogenic influences totalitarian and authoritarian regimes, cult of personality, radical religious schools and sects, modern information technologies, and so on.

\section{Results of the study and their discussion: Dependence on chemical and psychogenic psychoactive factors for the state of the same type}

It turned out that the dependence can be caused by exposure to any psychogenic external factor, if it resulted in the subject's strong, positive (from his/her point of view) emotions accompanied by bodily sensations. Indeed, in parties and sects with totalitarian ideology, personality cult, authoritarian regimes, under Nazism and racism psychogenic psychoactive influences are carried out. These effects are intended to deceiving into belief about the "selectness" of the adepts, the achievement of "perfection" or the utopian "bright future," the "outstanding" abilities of the head of the state, the "selectness" of the nation, the race. These beliefs lead to positive psychosomatic shifts in the individual, group or community of people: inspiration, placidity, winged sentiment, thuggish behavior, elation, etc., inducing long-term memory to form and remember the engrams of euphoria corresponding to the influencing psychoactive factor. Psychogenic psychoactive factors also can have a narcogenic effect. Some factors can cause a psychoemotional "splash" captured by long-term memory even after a single exposure, for instance, "love at the first sight" in love mania. Meanwhile, for the dependence on psychogenic factors with low narcogenicity - totalitarian political regimes, the personality cult - a prolonged ideological psychoactive influence is required. The rate of dependence development varies according to the reactivity of the autonomic nervous system: emotional people are more susceptible to various kinds of mania. People with reduced psychoemotional functionality ("emotionally greedy") and with the torpid psychoemotional sphere ("emotionally phlegmatic") are less susceptible to various dependences.

All this undoubtedly indicates that the dependences on psychogenic factors are the states of the same type as the dependences on PASs. To denote the whole variety of factors of chemical and psychogenic origin capable to cause dependence, the term "psychoactive factors" is acceptable [5] The psychoactive factor (PAF) is a psychogenic or chemical factor that can cause euphoria or other psychotropic effects desirable from the point of view of the consumer even after single exposure, and in a regular exposure they can cause psychological-psychic or psychological-psychophysical dependence.

\section{Differentiation of the states of progredient adaptive dependence of the body. Classification of psychoactive factors}

The principal difference between the state of progredient adaptation dependence and addiction consists in psychic stigmatization - the formation of stable neuronal engrams in the biological structures responsible for long-term memory,that affects further human behavior and creates a permanent psychic dependence on PAFs. But in addictions, processes occur at the psychological level, relatively stable conditional connections arise, and a relatively stable psychological dependence on the behavioral pattern arises - dependence on stereotypes of behavior, habits, ways of responding to some external psychogenic stimulus, etc. Although it is clear that in progredient adaptive dependences both on chemical and psychogenic PAFs, addictive behavior also takes place. Sometimes it is formed after the development of psychic dependence: when using heroin, at first a psychic dependence is formed, then the stereotype of the addict's behavior is formed. And in dependencies on chemical PASs with low narcogenicity - tobacco and alcohol psychological addiction, such as imitative smoking, alcoholic traditions - is formed at first, and then, in abuse, psychic and further psychophysical dependences are formed.

Under the influence of psychogenic PAFs capable of exerting a mass influence, such as the cult of personality, totalitarian regimes, one part of the society may develop a psychic dependence, while the other one only develops the psychological dependence - addictive behavior. It is necessary to differentiate the states of progredient adaptive dependence from the person's appetencies conditioned by endocrine diseases (i.e. bulimia - gluttony, polydipsia unquenchable thirst) and psychic illnesses when emotions caused by delusional ideas dominate in the psychoemotional sphere of a mentally ill person. Clinical identification of mental dependence on a PAS in a dependent person is possible.

\section{The Progression of The Hypermnesia Processes}

Feelings of positive psychophysical shifts under the influence of the PAF are fixed in memory. Such fixation is enhanced due to the regularity of PAF influence and the quantitative increase in the force of influence. That explains the stability of dependence on drug addiction, totalitarian political and religious ideologies, etc. The expansion of the memory engrams about positive psychophysical shifts is due to the memorization of concomitant stimuli accompanying the PAF influence. That explains the existence of rituals of joint smoking, alcohol consumption. All the mentioned above 
indicates the formation of adaptive, super-strong, expanded memory hyperengrams.

In PAF dependence changes in the biological mechanisms responsible for the euphoria hypermnesia are of a physiological, adaptive nature, and the available types of treatment consist only in blockade of the adaptation mechanisms against the repeated PAF influence. In this regard, euphoria hyperengrams remain saved in longterm memory and generally are the only cause of relapse. That is why a person dependent on illegal sexual activity can commit a sexual crime after having served long prison terms. Alcohol or drug dependent people after successful treatment and prolonged remission can resume alcohol and drug use. For love mania there is the expression "to fell in love for a lifetime" [6] Dependences on psychogenic PAFs (the cult of personality, totalitarian ideologies) persist up to the alternation of generation. The following studies are considered to be promising: studies of neurophysiological mechanisms of formation and fixation of euphoria engrams or other psychotropic effects desirable from the point of view of the consumer in the memory, development of methods of selective neutralization-"erasure from memory" of euphoria engrams (by patient's consent).

\section{Classification of psychoactive factors}

\section{Psychogenic PAFs}

a) Psychogenic PAFs capable of causing a mass adaptive psychological and psichic dependence: totalitarian and authoritarian political ideologies, the ideology of racism, Nazism, the cult of personality.

b) Psychogenic PAF capable of causing a group adaptation psychological and mental dependence - of religious and other totalitarian sects.

c) Psychogenic PAF - illegal actions capable of causing individual adaptation psychological and psychic dependence - dependence on sexual acts, prohibited by law, kleptomania, pyromania, etc. (all the abovementioned PAFs are prohibited or should be prohibited by law).

d) Other psychogenic PAFs (influences) capable of inducing an individual progredient adaptational psychological and psychic dependence - a state of falling in love, game addiction, etc.

\section{Chemical PAFs}

a) Chemical PAFs with high narcogenicity capable of inducing the accelerated development of individual progredient psychological and psychophysical adaptation dependence - drugs. (non-medical use and illegal trafficking are prohibited by law).

b) Chemical PAFs capable of inducing an individual progredient psychological and psychophysical adaptation dependence in case of abuse - alcohol, tobacco, etc.

Should other psychogenic and chemical PAFs dangerous to the individual and society are identified, they must be listed as prohibited by law.

\section{Maniology}

Narcology can only be a section of a new, broader branch of medicine that studies dependences both on chemical and psychogenic psychoactive factors. To denote a new branch, the term "maniology" is acceptable. (mania-propensity, appetency, Logos doctrine, i.e, the doctrine of propensities, appetencies).

Maniology is a branch of medical science that studies adaptogenesis and adaptive manifestations of the progredient adaptation psychic-psychological and psychological-psychophysical dependence of an individual, [7] a group of people and a society on various chemical and psychogenic psychoactive factors of the environment, medical, psychological, social and legal aspects of these problems, and develops methods of their prevention, treatment of deprivation syndrome and neutralization of appetency for the psychoactive factor.

In this regard, some definitions of various maniology sections, for example, narcology, should be changed.

Narcology is a section of maniology as a medical branch, which studies the adaptogenesis and adaptive manifestations of a person's progredient adaptation psychologicalpsychophysical dependence on various chemical psychoactive factors, the medical, psychological, social and legal aspects of these problems [8] and develops methods for their prevention, treatment of deprivation syndrome and neutralization of appetency for chemical psychoactive factor. To develop a new branch of medicine, there is a need in formation of Maniology scientific departments in higher medical schools.

\section{Conclusions}

a. Hypermnesia of external influences accompanied by strong emotional responses is an integral part of the evolutionary adaptive mechanism of the body.

b. The neurotransmitters released by the ANS in response to an external stimulus causing strong emotional responses are modulators of transmitting information from short-term to long-term memory.

c. The mechanism of preservation of psychoemotional 
sensations in long-term memory under the influence of psychoactive substances is identical to the evolutionary adaptive mechanism of hypermnesia.

d. Dependences of the body on psychoactive substances are the states of progredient adaptation.

e. Identity of the mechanisms of fixation of psychoemotional sensations in long-term memory under the influence of chemical and psychogenic psychoactive factors allows us to suggest that these states are states of the same type.

f. The presence of a psychic component in the appetency for any PAF is a distinctive feature of the state of adaptive progredient dependence on PAF, on the state of behavioral addiction and other appetencies.

g. Recommendations: It is necessary to create a new branch of medicine combining the study of human dependence both on chemical and psychogenic psychoactive factors.

Hypermnesia of euphoria or any other desired, from the point of view of the consumer, psychotropic effect as the initial and leading link in the occurrence and maintaining the body's dependence on psychoactive factors.

The work shows the role of the autonomic nervous system in functioning of long-term memory, the identity of functioning of the mechanisms of long-term memory in the evolutionary adaptation of a man and dependence on psychoactive substances. It is shown that the dependences of the body on psychoactive substances are the states of progredient adaptation, that the states of dependence of the body on psychoactive substances and on psychogenic psychoactive factors are the states of the same type. It is proposed to create a new branch of medicine combining study of the body's dependence both on chemical and psychogenic psychoactive factors. Classification of psychoactive factors is given. Onomastic definitions of terminology changes and additions to be used in this new branch of medicine are presented. Proposed allocation of the International Classification of Diseases is a separate chapter for the classification of states of progredient adaptation of the body depending on psychoactive factors.

\section{References}

1. AS Batuyev (1991) Higher nervous system. High School, Moscow, Russia p. $165-166,168-171$.

2. Baitubayev DG, Baitubayeva MD (2015) Physiology of progredient adaptation // Materials of 17th scientific and practical conference "Actual issues of psychiatry, narcology and medical psychology". NN Burdensome VSMA, Voronezh, Russia.

3. A Sh Zaichik LP, Churilov (2005) General pathophysiology. Publisher LLC "ELBI SPB", St.Petersburg, Russia, p. 23(26): 525.

4. AV Korobkov, AA Bashkirov, KT Vetchinkina (1980) Normal physiology Chapter 2. "Physiology of adaptation", Publisher "High School", Moscow, Russia, p. 494-499-503.

5. IN Pyatnitskaya Alcohol abuse and initial stage of alcoholism. Publisher "Medicine", Moscow, Russia. pp. 56-80.

6. IN Pyatnitskaya (1975) Clinical Drug Addiction. Publisher "Medicine", "Leningrad branch, Moscow, Russia, p. 3-6, 22-45, 47-55; 255-256, 261 $-263 ; 268-274$.

7. VM Smirnov, VN Yakovlev (2004) Physiology of the central nervous system. Publishing center Academy, Russia, p. 184-185; 250-251.

8. Kirilov OI, M Nauka (1994) "Stress hypertrophy of the adrenal glands" p.176. 